\title{
Effect of Paula exercise method on functional outcomes of women with post fistula repair incontinence: a protocol for randomized controlled trial
}

\author{
Saratu Umar Aliyu ${ }^{1,2}$, Shmaila M. Hanif ${ }^{3}$ and Isa Usman Lawal ${ }^{2^{*}}$ (1)
}

\begin{abstract}
Background: Post-fistula-repair incontinence (PFRI) is a common complication of vesicovaginal fistula (VVF) surgeries. It entails continuous leakage of urine after successful VVF closure. Pelvic Floor Muscle Training (PFMT) plays a vital role in the management of PFRI, however, an evolving exercise approach is the Paula Exercise Method (PEM) which has shown a promising effect in stopping urinary incontinence, but there is no data on its effect on PFRI. This study therefore, proposes to primarily investigate the effect of PEM on urine leakage and secondarily, pelvic floor strength (PFS), quality of life (QoL), sexual function (SF), and mental health (MH) in women with PFRI.
\end{abstract}

Methods: This is a study protocol for a randomized controlled trial. A total of 182 participants are expected to participate in the study after satisfying the inclusion criteria. The participants will be randomized into either PEM or PFMT study groups. The demographic data of all the participants will be recorded. Each participant will be assessed for urine leakage, PFS, QoL, SF, and MH at baseline and subsequently, at four, eight and 12 weeks of intervention.

Demographic parameters will be summarized using descriptive statistics. Continuous data will be computed for differences using inferential statistic of Analysis of variance, $t$-test and Man Whitney $\mathrm{U}$ as appropriate. All analyses will be performed using SPSS version 22.0 with probability set at 0.05 alpha level.

Discussion: It is hoped that the outcome of this study will determine the effect of the Paula exercise method on urine leakage, pelvic floor strength, quality of life, sexual function, and mental health among women with post-fistularepair incontinence and also provide evidence for the use of the Paula method in urinary incontinence.

Trial registration: Pan African Clinical Trials Registry (www.pactr.org), identifier PACTR201906515532827.

Keywords: Vesicovaginal fistula, Post repair incontinence, Urinary incontinence, Paula exercise method, Pelvic floor muscle training

\section{Background}

Vesicovaginal fistula (VVF) is an abnormal opening between the vagina and the bladder that occurs often

*Correspondence: iulawal.pth@buk.edu.ng

${ }^{2}$ Department of Physiotherapy, Faculty of Allied Health Sciences, College

of Health Sciences, Bayero University, Kano, Nigeria

Full list of author information is available at the end of the article during childbirth as a result of prolonged obstructed labour $[1,2]$. This occurs following compression on the pelvis by the foetal head resulting in tissue necrosis. This creates an opening between the vagina and bladder which leads to constant urine leakage from the vagina [1-3]. It is the major type of obstetric fistula, accounting for about $90 \%$ of all obstetric fistula cases $[1,4]$. It is also regarded as one of the major causes of morbidity among females in 
developing countries and some parts of developed countries $[2,5]$. The consequences of VVF can be devastating, as it affects women physically, socially, economically and psychologically $[3,4]$.

A number of studies have noted the risks for developing VVF as including: illiteracy, poverty or low socioeconomic status, childbearing at an early age, lack of access to antenatal care, lack of skilled or trained traditional birth attendants, and a malformed pelvis [3-8]. Obstetric VVF affects 2 million women worldwide, with a prevalence of 3 per 1000 women in sub-Saharan Africa $[2,5,8]$. In Nigeria, according to the Federal Ministry of Health [9], there are over 20,000 new cases of VVF yearly, in addition to the approximately 400,000-800,000 existing cases awaiting surgical repair. Over $85 \%$ of all cases of VVF are found in the north-western part of Nigeria [7, 10]; high incidences are reported in Kano, Katsina and Bauchi states in this region of the country [7].

Surgical interventions have been proven to be the effective management for VVF, especially in complex fistula, and in small fistula if conservative treatment fails $[1,11]$. Unfortunately, some women pass through the devastating period of having the VVF and undergoing the surgical procedure, but do not regain continence $[1,12,13]$. The VVF repair may be satisfactory anatomically, but inadequate functionally, as some women present with urinary leakage (called post-fistula repair incontinence) even after a successful repair [12-16].

Post-fistula repair incontinence (PFRI) as reported in the literature, is one of the most common complications of VVF surgeries, where urine continues to leak (especially with exertion) after successful VVF closure [12, 13, 17]. This incontinent gap is poorly understood and is a source of frustration to fistula surgeons (and particularly the patients) $[1,16,18-20]$.

Risk for developing PFRI depends on the site and type of fistula, as well as pelvic floor strength (PFS). Women with severe pelvic floor muscle (PFM) weakness are at risk of developing PFRI [21, 22]. According to Kayondo et al., type IIb fistulae are six times more likely to cause incontinence after successful repair than type I fistulae [22]. Also, women with circumferential fistula or larg fistulas are less likely to be continent after surgery [23]. PFRI also arises due to small bladder size/capacity following loss of the surface area of the bladder wall due to extensive fistula, neurologic damage, or due to the involvement of the urethral sphincter, especially if there is marked tissue loss and scarring $[1,8,12]$. Furthermore, PFRI occurs if there is loss of the sphincteric mechanism of the vesicourethral junction, and reduced bladder capacity or injury to the urethra $[14,20]$. It has been reported that the risk of PFRI is 50\% in women with minimal vaginal scarring and good bladder volume, while is $100 \%$ in women with reduced bladder volume and/or severe vaginal scarring [22-24]. Conservative interventions are the mainstay in the treatment of PFRI [11, 14, 20]; but unfortunately, supplementary surgery is prioritized over non-surgical options; the consequence of this is further deterioration of the condition [24]. It has however, been suggested that surgery should only be considered in persistent cases of PFRI or if the conservative treatment failed $[12,13]$.

Physiotherapy plays a vital role in the management of urinary incontinence (UI) and its associated symptoms through rehabilitation of the pelvic floor muscles (PFM), either directly or indirectly. Directly, the PFM can be rehabilitated through: pelvic floor muscle training (PFMT), biofeedback, cone therapy, and neuromuscular electrical stimulation [25, 26]; and indirectly through exercising other muscles of the body like the transverse abdominal muscles and circular or ring muscles (known as Paula Exercise Method), and also by patient education [27-29]. However, there is limited data on the role of physiotherapy on PFRI specifically. The available data mainly used pelvic floor muscles training (PFMT) $[8,13$, $14,17,30]$. And, to achieve positive effects of the PFMT, the exercise needs to be individualized and performed under professional supervision [21]. In addition, women find it difficult to voluntarily contract their PFM if it is weak even with proper instructions, and instead they end up contracting their abdominal, gluteal, and hip adductor muscles, and try to exaggerate inspiration which will often worsen their condition. Moreover, most of the VVF centres do not have physiotherapists or trained continent nurses to guide the women in performing the correct PFMT [23]. Even if there are professionals, teaching and monitoring the PFMT of patients individually may not be possible due to the high number of victims. Therefore, there is the need to find a conservative approach that can be performed with more ease and possibly in a group to accommodate the large number of the affected women and to encourage peer support, to help the affected women to get back to their normal life and minimize the need for another surgery.

The Paula exercise method also called circular/ring muscle exercise is a growing conservative method used in the treatment of urinary incontinence (UI) in general. It is an easy-to-perform approach, and has been tested to be effective in the management of UI and its associated complications in women without previous fistula repair [27, 29-31]. Practically, the Paula exercise method focuses on contracting and relaxing specific circular (or ring) muscles of the body in an effort to rehabilitate other damaged muscles of the body, because the body sphincters works together and their activity can affect one 
another due to oscillations in the spinal cord $[27,28,31$, 32].

The Paula method is considered an alternative to PFMT and has been found to exhibit comparable efficacy for urinary incontinence [31,33], including demonstrable long-term effects [34]. A study demonstrated comparable effectiveness of PFMT and PEM on sexual function and quality of life in women suffering from stress urinary incontinence [31]. However, a systematic review reported limited evidence for alternative exercise regimens for the reduction of urinary leakage in women with stress urinary incontinence [35]. The review found methodological limitations in PEM as an alternative to PFMT. The limitations included the fact that one of the studies was a pilot study [35] with small sample size while the other subjected the control, i.e. the PFMT group, to below-optimal training, and the high loss to follow-up (up to $28 \%$ ). Additionally, both studies were conducted by the same group of researchers. These factors obviously suggest the need for more studies with robust methodological approaches in diverse groups of researchers from distinct settings to investigate the efficacy of the Paula exercise method. This study aims to contribute to filling this gap and is therefore, targeted at investigating the effectiveness of the Paula exercise method in women with PFRI.

\section{Study objectives}

The main objective of this study is to investigate the effect of the Paula exercise method on urine leakage among women who suffered post-fistula repair incontinence (PFRI). In addition, the study will investigate the following:

1. Effect of the Paula exercise method on pelvic floor strength in women who suffered PFRI.
2. Effect of the Paula exercise method on quality of life of women who suffered PFRI.

3. Effect of the Paula exercise method on sexual function in women who suffered PFRI.

4. Effect of the Paula exercise method on mental health in women who suffered PFRI.

\section{Methods}

\section{Study design and sample}

The research design for this study is a randomized controlled trial (RCT). It is a quantitative research in the form of pretest, posttest and follow-up design. The study will be a two-arm, outcome assessor-blinded RCT. The two-arms will comprise an intervention and a control. Following participants' consent and baseline assessments, eligible participants shall be allocated randomly into either the intervention group (Paula exercise method) or the control group (pelvic floor muscle training). The proposed timeline for this study is 12 calendar months from participants' enrolment to follow-up assessments; Table 1 below:

\section{Setting}

All participants will be recruited from Murtala Muhammad Specialist Hospital (MMSH) and Jahun General Hospital (JGH) in Kano and Jigawa states of Nigeria respectively. The training will be conducted at the physiotherapy departments of the two institutions. The MMSH is a major health institution in Kano State; it is a specialist and referral centre to all hospitals in Kano State and its neighbouring states. It has a bed capacity of eight hundred and twenty six (826), thirty (30) wards and units, nine (9) operating theatres, fourteen (14) clinics, and a staff strength of 1656 . Similarly, JGH is a state hospital

Table 1 Study timeline from enrolment to completion

\begin{tabular}{|c|c|c|c|c|c|c|c|}
\hline \multirow[b]{2}{*}{ Time point } & \multirow{2}{*}{$\begin{array}{l}\text { Enrollment } \\
-\mathrm{t}\end{array}$} & \multirow{2}{*}{$\begin{array}{l}\text { Allocation } \\
0\end{array}$} & \multicolumn{4}{|c|}{ Post-allocation } & \multirow{2}{*}{$\begin{array}{l}\text { Follow-up } \\
\text { t4 (6 months) }\end{array}$} \\
\hline & & & t0 (0 week) & t1 (4 weeks) & t2 (8 weeks) & t3 (12 weeks) & \\
\hline \multirow{3}{*}{$\begin{array}{l}\text { Enrollment: eligibility screen informed consent alloca- } \\
\text { tion }\end{array}$} & $x$ & & & & & & \\
\hline & $x$ & & & & & & \\
\hline & & $x$ & & & & & \\
\hline \multirow{2}{*}{$\begin{array}{l}\text { Intervention: Paula exercise (experimental group) } \\
\text { Pelvic floor muscle training (control group) }\end{array}$} & & & $x$ & $x$ & $x$ & $x$ & \\
\hline & & & $x$ & $x$ & $x$ & $x$ & \\
\hline $\begin{array}{l}\text { Assessments: baseline variables_-age, weight, height, } \\
\text { blood pressure, marital status, educational status, } \\
\text { post fistula repair time }\end{array}$ & $x$ & $x$ & & & & & \\
\hline $\begin{array}{l}\text { Assessments: outcome variables } \\
\text { [Primary outcome- urine leakage] } \\
\text { [Secondary Outcome- pelvic floor strength, quality of } \\
\text { life, sexual function, depression and anxiety] }\end{array}$ & & & $x$ & $x$ & $x$ & $x$ & $x$ \\
\hline
\end{tabular}


located in Jahun local government area of Jigawa State. It provides quality health services to people in Jigawa State and the neighbouring states. It is a 300-bed capacity hospital with diverse clientele who receive treatment from the different clinical departments of the facility.

\section{Participants}

Participants in this study will comprise all women with PFRI attending the obstetrics and gynaecology departments of MMSH and JGH. However, only women who meet the study inclusion criteria will be screened and randomized into the experimental (Paula Exercise Method) and control (Pelvic Floor Muscles Training) groups. Participants will be screened for eligibility by the researcher and the research assistants. Participants will be considered eligible if: they have had the PFRI for more than 3 months, to ensure that healing has taken place $[1,14]$; they present with a $1 \mathrm{~g}$ gain in weight following a pad test; they consent to participate through a written informed consent; they are not above the age of 50 because as women age changes occur in the bladder and pelvic structures that can increase the risk of developing incontinence [36]; they have no faecal leakage; they have not had previous rectal surgery; they are not receiving any other alternative PFM rehabilitation at the point of recruitment; and urinary infection is ruled out because it affects the integrity of the pelvic organs [37].

After screening for eligibility, a cough test will be used to differentiate the type of incontinence. In the cough test, if urine leakage coincides with the cough, it is classified as stress incontinence (SI), however, if the leakage is delayed till after the cough, it is urge incontinence. No leakage after the cough signifies that it is overflow and functional incontinence [38].

\section{Sample size}

The sample size of this study was estimated based on the findings of a pilot study. We conducted a pilot study to test the adequacy and feasibility of this protocol as well as to determine the sample size required for this study. The pilot study participants were recruited after being certified as eligible to participate using the specified study inclusion criteria. Recruitment was conducted by reviewing records of the outpatient urology clinic on a weekly basis. A total of 30 women with post fistula repair incontinence participated in this pilot study. The 30 subjects were randomly assigned into the two arms of the study resulting into 15 participants in each arm of the pilot study (Paula Exercise Method and Pelvic Floor Muscle Training groups). All participants were assessed at baseline before randomization and prior to the commencement of intervention, post intervention (outcome).
The primary outcome of interest is Urine leakage. The programme was structured for 4-weeks of daily routine activities in both groups. The detail of urine leakage assessment is described later in the manuscript as the primary outcome measure.

In this pilot study the mean age of participants was $29.9 \pm 9.4$, the median Urine leakage score for PEM and PFMT was $4.53 \mathrm{~g}$ and $21.61 \mathrm{~g}$ respectively. We computed Mann Whitney $U$ for the data because the data was not normally distributed. Result for an independent two groups non-parametric test indicated significance $(U=38.00, p=0.020)$ with an effect size of $\eta^{2}=0.32$.

Using the outcome of the pilot study and according to a power calculation to detect a between-group difference, of 0.3 effect size for two independent means Mann Whitney $\mathrm{U}$ in urine leakage (UL) with $90 \%$ power at $\alpha=0.05$, a total sample size of 166 was generated. This suggests a total of 83 participants in each group. A $10 \%$ attrition rate will be considered, therefore, 182 participants will be recruited giving 91 participants in each group. The recruitment of participants from the two hospitals would be accomplished by: involving the surgeons from the departments of obstetrics and gynaecology of both hospitals who are involved in performing the fistula repairs (in the recruitment); and reviewing hospital registry and subsequently contacting patients whose records are available through individual phone contacts.

\section{Data collection procedure}

This study has been approved by the Human Research Ethics Committees of Kano State and Jigawa State Ministries of Health.

Assessment of all participants will be carried out in four stages (at baseline, at 4 weeks, at 8 weeks, and at the end of the study). A comprehensive assessment of the participants will be conducted, where personal demographic information (age, height, weight, marital status, educational status) and PFRI-specific information (time since VVF repair, type/size of the VVF, amount of urine leakage) will be assessed at baseline; and functional status information (QoL, MH, SF, and PFS) will be assessed at baseline and after every four weeks up to 12 weeks. All the parameters will be assessed during follow-up after 6 months.

\section{Randomization}

After screening the participants, and following baseline assessments, a computer-generated random allocation sequence will be used to assign the participants to either the intervention group (PEM group where they will receive PEM plus PFMT), or the control group (where they will receive PFMT only). To eliminate bias, the randomization will be carried out by a third party who is not 
aware of the study group. Assessment of outcome will be carried out by an experienced/trained researcher, who will be blinded to the type of intervention received by participants in each group. The study participants' flow diagram is presented in Fig. 1

\section{Interventions}

The interventions for the study will be PEM and PFMT. These training programmes will be administered by the researcher and four trained physiotherapists with postgraduate qualification in women's health. Two research assistants will be attached to each of the two training groups (i.e. two for PEM and two for PFMT). All trainings in both groups will be carried out in the morning. Both training programmes are structured to conform to the Consensus on Exercise Reporting Template (CERT) checklist $[39,40]$.

\section{The Paula exercise method}

The Paula exercise method will be adapted from previous studies where the method has been described in detail $[27,32]$. No equipment will be needed for the exercise. The exercise will involve contracting and relaxing the eye muscles daily. The participants will first assume a comfortable sitting position on a chair (with back rest), and relax for two (2) minutes. The researcher or research assistance will teach the participant (until mastered) how to effectively contract (squeeze) the eye muscles and hold it for five (5) seconds, and then relax the muscles for three (3) seconds (this makes one exercise cycle). The exercise cycle will be repeated forty (40) times in a daily session. The same exercise procedure will be repeated daily by all the participants for twelve (12) weeks. The exercise procedure will be performed under the supervision of the researcher or any of the two research assistants during Week One of the study. Subsequently, participants will be instructed to continue with the exercise procedure at

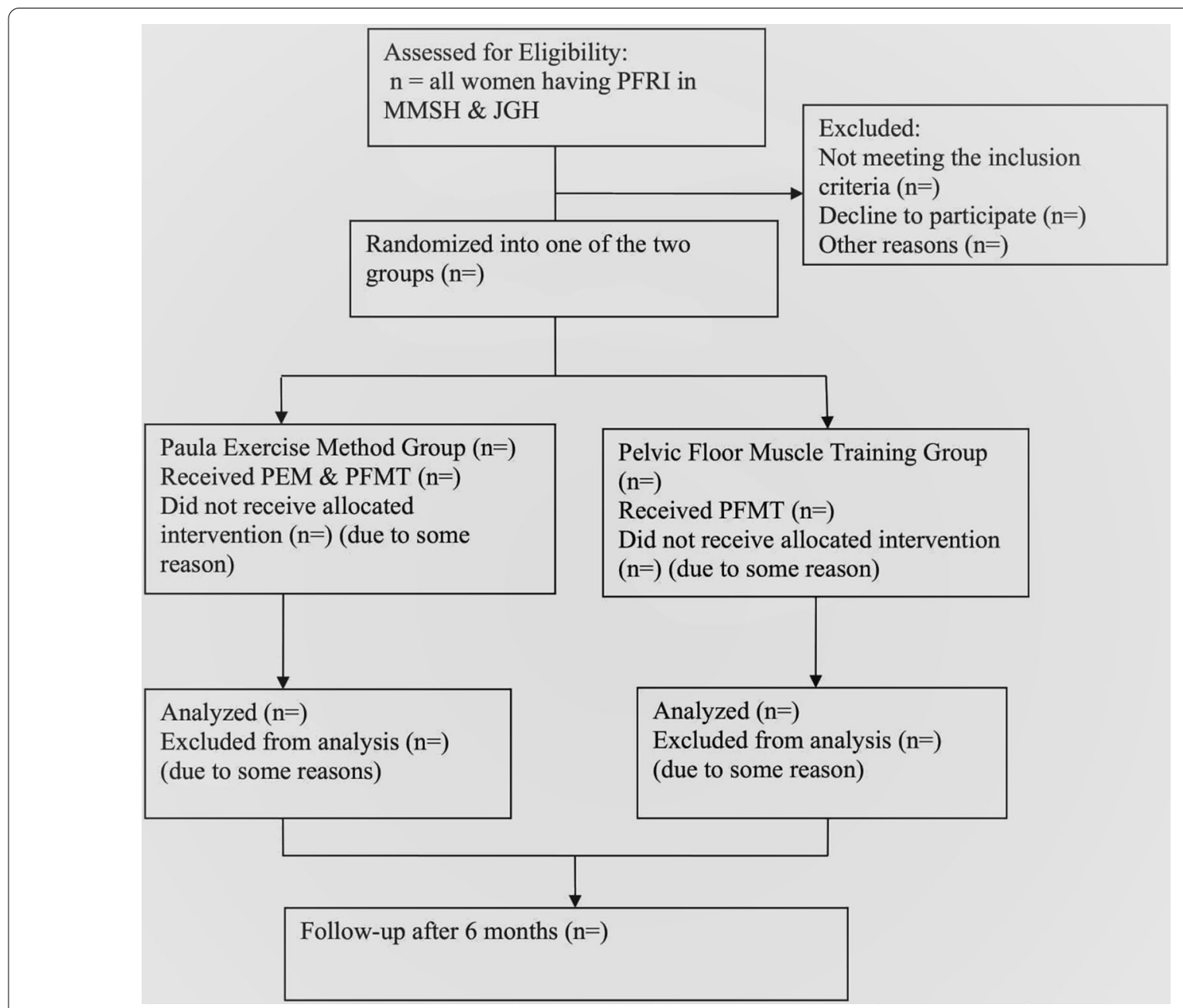

Fig. 1 Study flow diagram 
home for the remaining 11 weeks. Participants will each receive an activity log book in which to document their daily training activities (such as time training was performed and the duration of the activity) and any adverse event experienced during the training. The researcher and the research assistants will continually monitor the participants' daily exercise performances through phone calls to ensure adherence. At four, eight and twelve weeks, each participant will be asked to visit the study centres (physiotherapy departments of either MMSH or JGH according to where the participant was recruited) to perform the exercise in group (according to the protocol specific for this group) in the presence of the researcher and/or research assistant and to re-assess the functional outcomes.

\section{Pelvic floor muscle training (PFMT)}

The PFMT will be performed according to the clinical practice standard for pelvic floor exercise [27]. No equipment will be needed for the exercise procedure. The exercise will involve contracting (squeezing) and relaxing the PFM as if trying to stop urine from leaking or trying to raise the vagina up from the chair but, without contracting the abdominal, thigh, and buttock muscles. Each participant will be asked to comfortably lie supine on a bed (with knees in semi-flexed position) and relax for two (2) minutes before commencing the exercise. Each participant will receive detailed explanation on how to perform the exercise. Participants will then be asked to expose their vagina (to allow for visual assessment) and squeeze their PFM (as if trying to stop urination) and keep this position for a count of seven (7) and, then relax for a count of three (3). The researcher and/ or the research assistant will observe the contraction of the PFM to ensure that the exercise is done correctly. Participants will be asked to use a mirror to accomplish biofeedback for better understanding and accuracy of the procedure [41]. The exercise will be repeated until mastered by the participant. The exercise will then be performed in a sitting position where each participant will assume a comfortable sitting position on a chair (with back rest) for two (2) minutes, and then perform the exercise procedure as explained above. The exercise will be performed forty (40) times during a session once daily. Participants will perform the procedure individually in the study centre (of either at MMSH or JGH) for the first week and under the supervision of the researcher and/or two (2) research assistants. Subsequently, participants will be asked to perform the exercise at home; the researcher or research assistants will track the participants' individual daily exercise performance through phone calls to ensure adherence. Participants' homebased activities (time and duration of training performed and any adverse event experienced) will be recorded daily in the activity log book which will be given to each participant. At four, eight and twelve weeks, each participant will visit the study centre (of either MMSH or JGH) to perform the exercise in group (according to the protocol specific for this group) in the presence of the researcher and/or research assistant and to re-assess the functional outcomes.

In both study groups, a participant's treatment will be terminated/discontinued if the patient: (1) decides to opt out of the study, (2) develops persistent vaginal bleeding, (3) presents with other medical emergencies or adverse events including life-threatening episodes (uncontrollable bleeding); incapacitation; readmission into hospital or incidence of a sudden collapse resulting in a medical emergency within 48 -h would be rated as severe adverse episodes. However, minor adverse events would include bruise due to a scratch by exercise equipment, fall that does not result in loss of consciousness, fracture, admission into hospital, dyspnoea, cut, muscle soreness or pain persisting beyond $48 \mathrm{~h}$, and sudden hypertension or hypotension interfering with the study flow for a day. Participants' relations would be asked to document any such events within the period of the study and/or report such situation to the research team. Regular medical/surgical care being received by the patients, not involving exercise procedure of similar characteristics to this study exercise interventions would be allowed to continue for participants in both groups.

\section{Outcome}

A proforma will be developed by the researcher which will be used to document the demographic features (such as age, weight, height, blood pressure, address, type of incontinence, education status, and marital status) of all the participants as well as the pre, post and follow-up intervention findings of the study.

\section{Primary outcome measure}

The primary outcome measure is urine leakage, and it will be assessed using the $1-\mathrm{h}$ clinic pad test. The 1-h clinic pad test is an effective, simple, noninvasive, valid, and reliable method recommended by the International Continence Society for quantifying the amount of urine loss that will aid in the diagnosis/quantification of urinary incontinence, as well as assessing the efficacy of certain procedures that are used in the management of incontinence $[38,39]$. The procedure for the pad test will be in line with the recommendation of the International Continence Society. Each participant will be asked to empty their bladder before the test, and drink $500 \mathrm{ml}$ of water. A pre-weighed Always-Ultra pad will be given to the participant to place inside her underwear. The participant 
is then asked to sit for $15 \mathrm{~min}$. After this, the researcher will instruct the participant to cough 10 times, squat 10 times, jump up and down on the spot 10 times, and walk briskly for $10 \mathrm{~min}$. After 1-h, the pad will be removed and re-weighted using the Grams weighing scale. To quantify urine leakage, the difference in the pad weight will be calculated by subtracting the original pad weight from the pad weight after $1 \mathrm{~h}$. A $1 \mathrm{~g}$ gain in pad weight will be considered as proof of UI, and a reduction in urine leakage to $<1 \mathrm{~g}$ after the intervention will be considered as a cure.

\section{Secondary outcome measures}

The secondary outcome measures are Pelvic Floor Strength (PFS) to be measured using a perineometer; QoL to be measured using the Incontinence Quality of Life questionnaire; SF to be measured using the Female Sexual Function Index (FSFI); and Mental Health (MH) to be measured using the Hospital Anxiety and Depression rating scale (HADs). The perineometer is a highly reliable instrument used in assessing PFS with high intraclass correlation coefficient values of 0.95 and 0.88 for strength, and 0.94 and 0.83 for endurance, which indicate high within-day and between-days reliability for PFM strength and endurance respectively [42]. The PFS will be assessed according to standard protocol [42]. Each participant will be asked to lie supine with knees semiflexed; the probe of the perineometer will be inserted into the vagina until the full extent of the compressible portion of the device is at the level of the hymen. Each participant would then be instructed to maximally contract her PFM by trying to pull her pelvic floor in and up as much as possible (as if trying to stop urine from coming out) for $2-3 \mathrm{~s}$. The researcher will observe the participant to ensure that she is breathing normally. Three squeezes will be recorded at intervals of $10 \mathrm{~s}$ between the efforts, and the average will be recorded in $\mathrm{cms} / \mathrm{H}_{2} \mathrm{O}$ as the maximum perceived strength of the PFM; the higher the reading the higher the PFS [21].

The Incontinence QoL questionnaire is a valid and reliable questionnaire used to assess QoL with excellent internal consistency (Cronbach's value of 0.93 and $0.79-$ 0.89 ) and has no ceiling effect [43]. It is a 22 item selfreported disease-specific questionnaire that assesses the effect of UI on QoL under three (3) subscales of Avoidance and Limiting Behaviour (ALB), Psychosocial Impact (PSI), and Social Embarrassment (SE) [28, 43]. Specifically, the ALB subscale consists of 8 items, the PSI consists of 9 items, and the SE consists of 5 items. Moreover, scores ranging from 1 (extreme) to 5 (not at all) will be used to grade each of the items, and the total scores (for the 22 items) will then be transformed to a "scale score" ranging from 0 to 100 using the following formula: $\begin{aligned} \text { Scale score }= & \text { Sum of the items }- \text { Lowest possible } \\ & \text { score } / \text { Possible raw score range } \times 100 .\end{aligned}$

Higher scores from all the items indicate less impact of PFRI on QoL [28, 43].

The Female Sexual Function Index (FSFI) is a questionnaire used in the assessment of female sexual function. The questionnaire possesses good psychometric properties (high test-retest reliability for each domain $\mathrm{r}=0.79$ to 0.86 , and high internal consistency (Cronbach's values of 0.82 and above) and it is easy to administer [44]. It is a multi-dimensional questionnaire with 19 items that are self-reported for assessing SF in women. It comprises six (6) domains: desire, subjective arousal, lubrication, orgasm, satisfaction, and pain. The desire domain has 2 items with scores ranging from 1 to 5 in each item, summed up to a total score of 2 (minimum) and 10 (maximum); the arousal domain has 4 items with scores ranging from 0 to 5 in each item, summed up to a total of minimum and maximum scores of 0 and 20 respectively; the lubrication domain has 4 items with scores ranging from 0 to 5 in each item, summed up to a total of minimum and maximum scores of 0 and 20 respectively; the orgasm domain has 3 items with scores ranging from 0 to 5 in each item, summed up to a total of minimum and maximum scores of 0 and 15 respectively; the satisfaction domain has 3 items with scores ranging from 1 to 5 in each item, summed up to a minimum and maximum score of 2 and 15 respectively; and the pain domain has 3 items with scores ranging from 0 to 5 in each item, summed up to a total of minimum and maximum score of 0 and 15 respectively. An FSFI total score of less than or equal to 26.55 indicates risk for sexual dysfunction.

The Hospital Anxiety and Depression rating scale (HADs) will be used to assess mental health (MH). It is a valid and reliable questionnaire used in determining the mental status of an individual [45]. Each participant will be asked to complete the HADs after clear and detailed explanation by the researcher on how to complete the questionnaire. The HADs comprises seven questions for anxiety and seven for depression and it takes $2-5$ min to complete. The anxiety and depression questions are combined in the scale; however, it is recommended that they are scored separately [46]. Both scales demonstrate good to moderate to high internal consistency; Cronbach's alpha for anxiety varied from 0.68 to 0.93 (mean 0.83 ) and for depression from 0.67 to 0.90 (mean.82) [45].

\section{Data analysis}

Microsoft Excel will be used to record all the data obtained before being exported to the Statistical Package for Social Sciences (SPSS). Descriptive and inferential statistics will be used to analyse the data. All study 
parameters will be summarized using descriptive statistics of frequency, mean, standard deviation, and percentage. Factorial ANOVA will be used to determine between-group differences/interactions in the study groups from the pre to the follow-up study period for PFS and UL among study participants. The Wilcoxon signed-rank test will be computed to determine differences between the pre, post and follow-up intervention for SF, ML and QoL scores in both the experimental and control groups. The independent $t$-test will be used to determine the difference in the post intervention PFS and UL scores between the experimental and control groups, while Mann-Whitney $U$ will be used to determine the difference in the post intervention for SF, ML and QoL scores between the experimental and control groups. The magnitude of the differences between the groups will be determined by calculating the effect sizes. Missing data will be handled through multiple imputation. All analyses will be performed with probability set at 0.05 alpha level.

\section{Discussion}

The Paula exercise method is a growing conservative method used in the management of urinary incontinence with significant effect in reducing urine leakage and improving pelvic floor muscles strength, QoL and SF as reported by some studies $[23,31,33]$. However, this intervention has not been tested on women with postfistula-repair incontinence and the evidence regarding the use of alternative exercise other than PFMT for UI has been sketchy. Women with PFRI are devastated and many continence surgeries only worsen their conditions [28]. Therefore, there is an urgent need for investigation of other conservative approaches that may help the affected women to re-integrate back into the society.

The outcome of this study will provide evidence on whether PEM can be used in the management of PFRI. Also, it will further support the use of Paula exercise as a conservative treatment for UI. Findings shall be disseminated appropriately at relevant scientific forums.

\section{Abbreviations \\ VVF: Vesicovaginal fistula; PFRI: Post fistula repair incontinence; PFS: Pelvic floor strength; UI: Urinary incontinence; PFM: Pelvic floor strength; PFMT: Pelvic floor strength training; PEM: Paula exercise method; UL: Urine leakage; QoL: Quality of life; MH: Mental health; SF: Sexual function; FSFI: Female Sexual Function Index; HADs: Hospital Anxiety and Depression Rating Scale; ALB: Avoidance and limiting behavior; PSI: Psychosocial impact; SE: Social embarrassment.}

\section{Acknowledgements}

We appreciate the contribution of Dr. Mukadas O. Akindele and Dr. Bashir Bello, both of the Department of Physiotherapy, Faculty of Allied Health Sciences, College of Health Sciences, Bayero University, Kano, Nigeria, for reviewing the primary proposal of this study.

\section{Authors' contributions}

SUA and SMH contributed to the development of the study concept and design, SMH, SUA and IUL were responsible for development of the research question and the study protocol. SUA and SMH arranged and prepared the exercise interventions. SUA drafted the preliminary manuscript; IUL prepared the final manuscript and its editing. All authors discussed the study design and contributed to the protocol. All authors have read and approved the final version of this manuscript.

\section{Funding}

No funding has been received for this study.

Availability of data and materials

The data for this trial will be made available upon request.

\section{Declarations}

\section{Ethics approval and consent to participate}

This study protocol has been fully approved by the ethics committees of the Kano (MOH/Off/797/T.I/518) and Jigawa (MOH/SEC.3/S/829/I) States Ministries of Health. Trial registration for this protocol was obtained from Pan African Clinical Trials Registry (PACTR201906515532827). Written informed consent of each eligible participant shall be sought by the recruiting researcher along with the surgeons (where applicable). All data collected will be upheld with utmost confidentiality, individual names of participants is not needed and as such will not be collected.

\section{Consent for publication}

Not presently applicable.

\section{Competing interests}

The authors declare that they have no conflict of interest.

\section{Author details}

1 Department of Physiotherapy, Rasheed Shekoni Teaching Hospital, Dutse, Jigawa State, Nigeria. ${ }^{2}$ Department of Physiotherapy, Faculty of Allied Health Sciences, College of Health Sciences, Bayero University, Kano, Nigeria. ${ }^{3}$ Department of Health Professions, Faculty of Health, Psychology and Social Care, Manchester Metropolitan University, Manchester, England.

Received: 12 July 2020 Accepted: 1 March 2021

Published online: 09 March 2021

\section{References}

1. Kopp DM, Bengtson AM, Tang JH, Chipungu E, Moyo M, Wilkinson J. Use of a postoperative pad test to identify continence status in women after obstetric vesicovaginal fistula repair: a prospective cohort study. BJOG Int J Obst Gynaecol. 2017;124(6):966-72.

2. Drew LB, Wilkinson JP, Nundwe W, Moyo M, Mataya R, Mwale M, Tang JH. Long-term outcomes for women after obstetric fistula repair in Lilongwe, Malawi: a qualitative study. BMC Pregnancy Childbirth. 2016;16(1):2.

3. Kimani ZM, Ogutu O, Kibe A. The prevalence and impact of obstetric fistula on women of Kaptembwa, Nakuru Kenya. Int J Appl Sci Technol. 2014;4(3):273.

4. Lawani LO, Iyoke CA, Ezeonu PO. Contraceptive practice after surgical repair of obstetric fistula in southeast Nigeria. Int J Gynecol Obstet. 2015;129(3):256-9.

5. Umoiyoho AJ, Inyang-Etoh EC. Community misconception about the aetiopathogenesis and treatment of vesicovaginal fistula in northern Nigeria. Int J Med Biomed Res. 2012;1(3):193-8.

6. Ahmed ZD, Abdullahi HM, Yola Al, Yakasai IA. Obstetrics fistula repairs in Kano, Northern Nigeria: the journey so far. Ann Trop Med Public Health. 2013;6(5):545.

7. Edeh SB. 12,000 women develop VVF every year in Nigeria. On July 20, 2012. In: News Vanguard; 2012. 
8. Stamatakos M, Sargedi C, Stasinou T, Kontzoglou K. Vesicovaginal fistula: diagnosis and management. Indian J Surg. 2014;76(2):131-6.

9. Federal Republic of Nigeria, Ministry of Health. Saving newborn lives in Nigeria: Newborn health in the context of the Integrated Maternal, Newborn and Child Health Strategy. Abuja: Federal Ministry of Health, Save the Children, ACCESS; 2009 (www.savethechildren.org/savenewborns).

10. Kuti $\mathrm{O}$. The Girl, bringing her out of the shadow of tomorrow's Woman Population information and Communication Bureau Federal Ministry of Information, 2001

11. Raheem AA, Zoaier A, El-Sherbiny A. Childbirth and lower urinary tract complications. In: Childbirth trauma., London: Springer; 2017. p. 151-179.

12. Goh JT, Krause H, Tessema AB, Abraha G. Urinary symptoms and urodynamics following obstetric genitourinary fistula repair. Int Urogynecol J. 2013;24(6):947-51.

13. Krause HG, Lussy JP, Goh JT. Use of periurethral injections of polyacrylamide hydrogel for treating post-vesicovaginal fistula closure urinary stress incontinence. J Obstet Gynaecol Res. 2014;40(2):521-5.

14. Browning A. A new technique for the surgical management of urinary incontinence after obstetric fistula repair. BJOG Int J Obstet Gynaecol. 2006:113(4):475-8.

15. Browning A, Menber B. Women with obstetric fistula in Ethiopia: a 6-month follow up after surgical treatment. BJOG Int J Obstet Gynaecol. 2008;115(12):1564-9.

16. Keyser L, McKinney J, Salmon C, Furaha C, Kinsindja R, Benfield N. Analysis of a pilot program to implement physical therapy for women with gynecologic fistula in the Democratic Republic of Congo. Int J Gynecol Obstet. 2014;127(2):127-31.

17. Government of Nepal Ministry of Health and Population National Health Training Center. Management of Obstetric Fistula for Health Care Providers-On-the-Job Training. Jhpiego; 2015. 117 p https://www.glowm .com/resources/glowm/pdf/POFS/POFS_Chap_09.pdf.

18. Hancock B, Browning A. Practical obstetric fistula surgery. London: Royal Soc. of Medicine Press; 2009.

19. Carey MP, Goh JT, Fynes MM, Murray CJ. Stress urinary incontinence after delayed primary closure of genitourinary fistula: a technique for surgical management. Am J Obstet Gynecol. 2002;186(5):948-53.

20. Iancu G. Obstetric fistula. In: Childbirth trauma; 2017. London: Springer, p. 231-7.

21. Talasz H, Himmer-Perschak G, Marth E, Fischer-Colbrie J, Hoefner E, Lechleitner M. Evaluation of pelvic floor muscle function in a random group of adult women in Austria. Int Urogynecol J. 2008;19(1):131-5.

22. Kayondo M, Wasswa S, Kabakyenga J, Mukiibi N, Senkungu J, Stenson A Mukasa P. Predictors and outcome of surgical repair of obstetric fistula at a regional referral hospital, Mbarara, western Uganda. BMC Urol. 2011;11(1):23.

23. Goh JT, Krause H. Urinary incontinence following obstetric fistula repair. World J Obstet Gynecol. 2016;2:5.

24. Browning A. Obstetric fistula in Ilorin, Nigeria. PLOS Med. 2004;1(1):e2.

25. Bø K. Pelvic floor muscle training in treatment of female stress urinary incontinence, pelvic organ prolapse and sexual dysfunction. World J Urol. 2012;30(4):437-43.

26. Browning A. Prevention of residual urinary incontinence following successful repair of obstetric vesico-vaginal fistula using a fibro-muscular sling. BJOG Int J Obstet Gynaecol. 2004;111(4):357-61.

27. Liebergall-Wischnitzer M, Paltiel O, Celnikier DH, Lavy Y, Manor O, Wruble AC. Sexual function and quality of life of women with stress urinary incontinence: a randomized controlled trial comparing the Paula method (circular muscle exercises) to pelvic floor muscle training (PFMT) exercises. J Sex Med. 2012;9(6):1613-23.

28. Shelly B. Evidenced-based physical therapy for the pelvic floor: bridging science and clinical practice. J Women's Health Phys Ther. 2008;32(1):37.

29. Tajiri K, Huo M, Maruyama $\mathrm{H}$. Effects of co-contraction of both transverse abdominal muscle and pelvic floor muscle exercises for stress urinary incontinence: a randomized controlled trial. J Phys Ther Sci. 2014;26(8):1161-3.

30. Tayler-Smith K, Zachariah R, Manzi M, van den Boogaard W, Vandeborne A, Bishinga A, De Plecker E, Lambert V, Christiaens B, Sinabajije G, Trelles M. Obstetric fistula in burundi: a comprehensive approach to managing women with this neglected disease. BMC Pregnancy Childbirth 2013;13(1):164

31. Liebergall-Wischnitzer M, Hochner-Celnikier D, Lavy Y, Manor O, Shveiky D, Paltiel O. Randomized trial of circular muscle versus pelvic floor training for stress urinary incontinence in women. J Womens Health. 2009;18(3):377-85.

32. Garbourg, P. The secret of the ring muscles: healing yourself through sphincter exercise. Wayne, United States: Avery Publishing Group; 1997. Print.

33. Liebergall-Wischnitzer M, Hochner-Celnikier D, Lavy Y, Manor O, Arbel $\mathrm{R}$, Paltiel O. Paula method of circular muscle exercises for urinary stress incontinence-a clinical trial. Int Urogynecol J. 2005;16(5):345-51.

34. Liebergall-Wischnitzer M, Paltiel O, Lavy Y, Shveiky D, Manor O, HochnerCelnikier D. Long-term efficacy of Paula method as compared with pelvic floor muscle training for stress urinary incontinence in women: a 6-month follow-up. J Wound Ostomy Continence Nurs. 2013;40(1):90-6.

35. $\mathrm{B} \varnothing \mathrm{K}, \mathrm{Herbert} \mathrm{RD}$. There is not yet strong evidence that exercise regimens other than pelvic floor muscle training can reduce stress urinary incontinence in women: a systematic review. J Physiother. 2013;59(3):159-68.

36. Abrams P, Cardozo L, Khoury S, Wein AJ, editors. Incontinence: 2013. International Consultation on Urological Diseases; 2013.

37. Hakim LS, DaSilva GM. Female sexual dysfunction. In: Pelvic floor dysfunction; 2006. London: Springer, p. 97-105.

38. Khandelwal C, Kistler C. Diagnosis of urinary incontinence. Am Fam Phys. 2013;87(8):543-50.

39. Peterson AC, Amundsen CL, Webster GD. The 1-hour pad test is a valuable tool in the initial evaluation of women with urinary incontinence. Female Pelvic Med Reconstr Surg. 2005;11(5):251-6.

40. Slade SC, Dionne CE, Underwood M, Buchbinder R. Consensus on Exercise Reporting Template (CERT): explanation and elaboration statement. Br J Sports Med. 2016;50(23):1428-37.

41. Oliveira M, Ferreira M, Azevedo MJ, Firmino-Machado J, Santos PC. Pelvic floor training protocol for stress urinary incontinence in women: a systematic review. Rev Assoc Med Bras. 2017;63(7):642-50.

42. Rahmani N, Mohseni-Bandpei MA. Application of perineometer in the assessment of pelvic floor muscle strength and endurance: a reliability study. J Bodyw Mov Ther. 2011;15(2):209-14.

43. Schurch B, Denys P, Kozma CM, Reese PR, Slaton T, Barron R. Reliability and validity of the Incontinence quality of life questionnaire in patients with neurogenic urinary incontinence. Arch Phys Med Rehabil. 2007;88(5):646-52.

44. Rosen C, Brown J, Heiman S, Leiblum C, Meston R, Shabsigh D, Ferguson RD. The Female Sexual Function Index (FSFI): a multidimensional selfreport instrument for the assessment of female sexual function. J Sex Marital Ther. 2000;26(2):191-208.

45. Bjelland I, Dahl AA, Haug TT, Neckelmann D. The validity of the Hospital Anxiety and Depression Scale: an updated literature review. J Psychosom Res. 2002;52(2):69-77.

46. Stern AF. The hospital anxiety and depression scale. Occup Med. 2014:64(5):393-4.

\section{Publisher's Note}

Springer Nature remains neutral with regard to jurisdictional claims in published maps and institutional affiliations. 